\title{
ONE-VALUED MAPPINGS OF GROUPS INTO FIELDS
}

\author{
KATSUHIKO MASUDA
}

The aim of this article is to investigate algebraic nature of systems of onevalued mappings of given group into given field and to apply it to the theory of Galois algebras and duality of compact $T_{n}$-groups. The results obtained in the following are those; factor systems of Galois algebras with finite Galois groups are defined without any restrictions on the orders of Calois groups and the coefficient fields, a necessary and sufficient condition for them to be associated with Galois fields is obtained, dualities of finite groups are obtained very simply without any restrictions for coefficient field of representations, and Tannaka's duality ${ }^{11}$ of compact $T_{0}$-groups is proved without the use of the compactness of Tannaka representation groups ${ }^{2)}$ of representations of compact $T$, groups and the use of Kampen's theorem. ${ }^{3)}$

$\S 1$. One-valued mappings of a group into a field.

1. Let $G$ be an arbitrary, not necessarily finite. group, $\Omega$ be an arbitrary field. Let $\&$ denote the group ring $G(\Omega)$ of $G$ over $\Omega$, and $M$ denote the $(S$ module which consists of all one-valued mappings of $G$ in $\Omega$, having the addition and the operation of $G$ defined by:

$$
f(\sigma)+g(\sigma)=f+g(\sigma), \quad f^{\prime}(\sigma)=f(\sigma)
$$

for $f, g \in \mathfrak{M}$ and $\sigma, \tau \in G$. If we define a distributive multiplication in $\mathfrak{l}_{\mathrm{f}}$ to make it an algebra, not necessarily associative, over $\Omega$ and if the operation of $G$ defined above for $\mathfrak{M}$ gives automorphisms of the thus obtained algebra, we call it a Galois algebra over $\Omega$ with Galois group $G$. We call an algebra $K$, not necessarily associative, over $\Omega$ with $G$ as operator domain also Galois algebra over $\Omega$ with Galois group $G$, if and only if there exists at least a $G$-permissible ring-isomorphic mapping on $K$ from one of Galois algebras obtained from $M$. When $G$ is finite, this definition is clearly equivalent with T. Nakayama s."

Received September 12, 1952.

1) T. Tannaka, Über den Dualitätssatz der nicht kommutativen topologische Gruppe, Tohoku Math. J. 45 (1938).

M. Krein, On the almost periodic functions on a topological group. C. R. (Doklady) 30 (1941).

2) Cf. Tannaka's above article.

3. E. R. van Kampen, Almost periodic functions and compact groups, Annals of Math. 37 (1936).

1) T. Nakayama, Construction and characterization of Calois algebras with given Galois group. Nagoya Math. J. 1 (1950). 
We denote different Galois algebras obtained from the same $\mathfrak{M}$ with different multiplications by different symbols. The ordinary multiplication as functions, defined by

$$
f(\sigma) g(\sigma)=f g(\sigma)
$$

( $f . g \in \mathfrak{M} ; \sigma \in G$ ), satisfies clearly the above conditions, and makes $\mathfrak{M}$ a certain Galois algebra. We denote it by $(5$. Let $D$ be an arbitrary representation of $G$ by regular matrices in $\Omega$ of degree $f, D(\sigma)$ denote the matrix corresponding to $\sigma$ by the representation $D$. The $i j$-element of the representing matrix is naturally a one-valued mapping $d_{i j}$ of $G$ in $\Omega$, and we denote by $d_{i j}^{\prime}$ the element of $\mathfrak{D}$ which maps each $\sigma \in G$ to $d_{i j}\left(\sigma^{-1}\right)$, by $D^{\prime}$ the matrix $\left(d_{i j}^{\prime}\right)_{i j}$.

2. As $[$ is clearly associative and commutative, we can apply the theory of determinants to the matrices in (F, and obtain

Lemma 1. Considered as a matrix in (5) of degree $f, D^{\prime}$ is regular.

Proof. The determinant $\left|D^{\prime}\right|$ of the matrix $D^{\prime}$ is the mapping of $G$ in $\Omega$ which maps each element $\sigma$ of $G$ on the determinant $\left|D\left(\sigma^{-1}\right)\right|$ of the regular matrix $D\left(\sigma^{-1}\right)$ in $\Omega$ of degree $f$, and is clearly a regular element of 5 .

From Lemma 1 we obtain easily the following Lemma 2 and its corollary, which are fundamental in the theory of Galois algebras.

Lemma 2. Let $A$ be an arbitrary square matrix in $\mathfrak{R}$ of degree $f$, such that

$$
A^{\sigma}=D(\sigma) A \text { for each } \sigma \in G \text {. }
$$

Then there exists one and only one, not necessarily regular, square matrix $C$ in $\Omega$ of degree $f$, such that

$$
A=D^{\prime} C .
$$

Proof. As $\mathbb{E}$ is as $\mathfrak{B}$-module $\mathfrak{M}$ itself, we can consider $A$ as a matrix in $\mathfrak{F}$. The assertion follows then easily from Lemma 1.

Corollary. Let $B$ be an arbitrary square matrix of degree $f$ in an arbitrary Galois algebra $K$ over $\Omega$ with Galois group $G$, such that

$$
B^{\sigma}=D(\sigma) B \text { for each } \sigma \in G \text {. }
$$

Then there exists one and only one matrix $C$ in $\Omega$ such that

$$
B=D^{\mu} C \text {, }
$$

where $/$ denotes an isomorphic mapping on $K$ of a certain Galois algebra obrained from $\mathfrak{M}$.

3. From now on we suppose always that there exist sufficiently many representations of $G$ with regular matrices in $\Omega$, that is, for each pair of different 
e'ements $\sigma$, $\tau$ of $G$ there exists at least a representation $D$ of $G$ with regular matrices in $\Omega$ such that $D(\sigma) \neq D(\tau)$. We call a system $\left\{D_{k} ; \% \in X\right\}$ of representacions of $G$ with regular matrices in $\Omega$ a basic system of representations of $G$ in $\Omega$, when for each two representation $D_{.}, D_{\%}$ in the system there exists aí least a regular matrix $P_{\%, \psi}$ in $\Omega$ of degree $f_{f} f_{\psi}$ such that

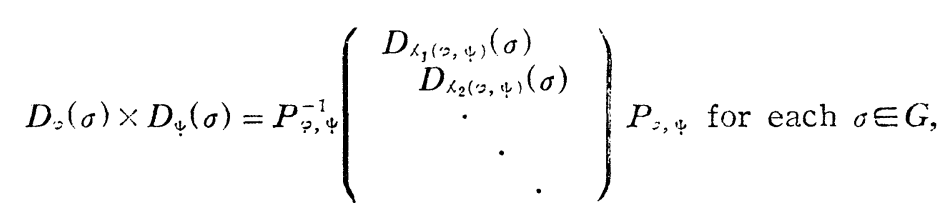

where $D_{i_{i}(a, 4)}$ 's are representations of $G$ in the system, and arbitrary two representations in the system with different suffices are inequivalent.

Now let $\Gamma$ denote a basic system $\left\{D_{\%} ; \% \in X\right\}$ of representations of $G$ in $\Omega$. From Corollary to Lemma 2 follows easily that for each two representations $D ., D_{\psi}$ of $\Gamma$ there exists one and only one matrix $C_{p, y}$ in $\Omega$ of degree $f_{:} f_{y}$. such that

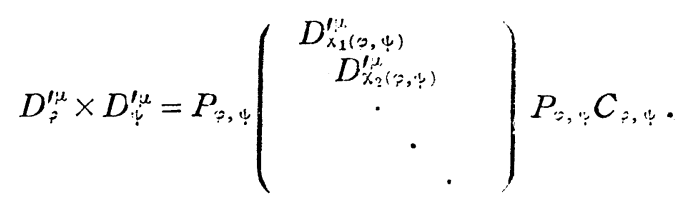

We call the system $\{C, \varphi ; \varphi, \phi \in X\}$ a facior sys'em asscciated with $K$ in reference to $I$. From the existence of such marrices $C_{r, y}$ in $\Omega$ follows that the moduie $T_{K}$ generated in $K$ over $Q$ by all the coefficients of matrices $D^{\prime \prime-\alpha} s$ with $D \in I$ is a $G$-permissible subalgebra of $K$, and from Corollary to Lemma 2 follows that $\mathscr{D}_{K}$ is determined uniquely by $r$, independent of the choice of the isomorphism $\mu$.

$\S 2$. Galois algebras with finite Galois groups.

4. We suppose throughout $\$ 2$ that $G$ is a finite group, accordingly our Galois algebra $K$ over $\Omega$ with Galois group $G$ is of finite rank over $\varrho$, and that $\Gamma=\left\{D_{k} ; \% \in X\right\}$ consists of ail distinct indecompusable constituents of the regular representation of $G$ over $\Omega^{5)}$ Then $\mathscr{D}_{K}$ coincicies with $K$ itself. For, if we decompose $K$ into a direct sum of indecomposable (\$)-submodules, then by the Corollary to Lemma 2 any basis of such a $\$ S$-submodule of $K$ lies in $I_{k}$. and

$$
\bar{K}=\mathfrak{T}_{K^{*}} \text {. }
$$

So the multiplication of a Galois algebra $K$ of finite rank is uniquely determined. if a factor system associated with in reference to $\Gamma$ is given. Now

5) Cf. M. Osima, Note on the Kronecker product of representations of group, Proc. Acad. Japan 17 (1941). 
we set to each pair of elements $\varphi, \psi$ of $X$ arbitrary square matrix $C_{\hat{\gamma}, \psi}$ in $\Omega$ of degree $f_{f} f_{\psi}$. It is easy to see that the thus constructed system $\left\{C_{\hat{\gamma}, \varphi} ; \varphi\right.$, $\psi \in X\rangle$ is a factor system, if and only if for every matrix $M \%$ in $Q$ or degree $f_{\%}$ satisfying

$$
\operatorname{Spur}\left(D_{\varkappa} M_{\varkappa}\right)=0
$$

holds

$$
\begin{array}{r}
\operatorname{Spur}\left(D_{\varkappa}(\sigma) \times D_{\hookrightarrow}(\sigma) \cdot C_{\varkappa, \varphi} \cdot M_{\%} \times E_{f_{\psi}}\right)=0 \quad \text { for each } \quad \phi \in X, \\
\text { each } \sigma \in G,
\end{array}
$$

where we denote by $E_{f_{\psi}}$ the unit matrix in $\Omega$ of degree $f_{\psi}$.

5. We can obtain conditions for factor systems to be associated with associative, commutative, associative and commutative, or associative, commutative and absolutely semisimple ${ }^{6)}$ Galois algebras and the condition for two factor systems to be equivalent, that is, to be associated with isomorphic Galois algebras over $\Omega$ with Galois group $G$, like in H. Hasse's article "Invariante Kennzeichnung Galoisscher Körper mit vorgegebener Galoisgruppe" ") (quoted as H. I. in the following). Here we discuss only the condition for commutative and associative factor systems to be associated with semisimple Galois algebras. We define "spur" of an element of Galois algebra $K$ of finite rank by

$$
S(w)=\sum_{\sigma \in G} w^{\sigma} \quad \text { for each } \quad w \in K .
$$

Discriminants, constructed with reference to above defined "spur," of a commutative Galois algebra clearly becomes 0 , if it has radical. The converse follows from the fact that an associative, commutative and semisimple Galois algebra is a direct sum of separable Galois fields ${ }^{8)}$ and each discriminant of it has discriminants of component separable Galois fields as its components. If a factor system is given, discriminants defined above can be calculated from

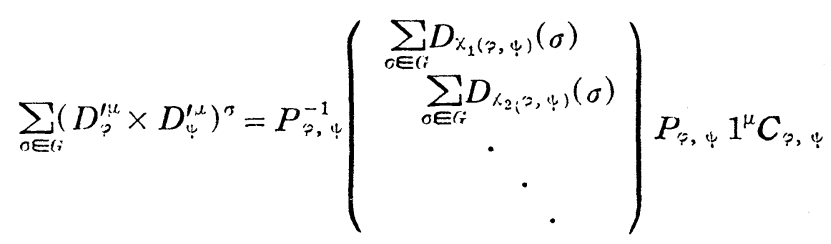

6) An asscciative, commutative, and semisimple Galois algebra is necessarily absolutely semisimple; cf. H. I.

$\therefore$ H. Hasse, J. reine angew. Math. 187 (1950).

$\therefore$ Cf. ${ }^{6}$. 


$$
=P_{p, \varphi}^{-1}\left(\begin{array}{c}
D_{\chi_{1}(\gamma, \varphi)}(G) \\
D_{\gamma_{2}(=, 4)}(G) \\
\cdot \\
\cdot
\end{array}\right) P_{\gamma, \varphi} C_{1,1} C_{p, \varphi},
$$

where 1 denotes the unit character of $G$ in $\Omega$.

Though the above considerations lead to most general results, it does not yet enable us to find independent parameters of the manifold of all factor systems of $G$ in $\Omega$ nor to formulate above conditions explicitly in reference to them. When absolutely irreducible representations of $G$ are obtained in $\Omega, T$. Nakayama's results in his article "On construction and characterization of Galois

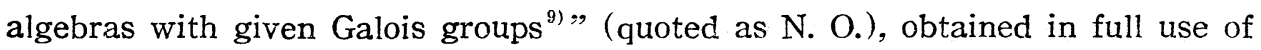
the theory of modular regular representations, afford a complete answer to it.

6. Let $H$ be a subgroup of $G$. We say that a factor system $\left\{C_{p, 4} ; \varphi\right.$, $\psi \in X\}$ of $G$ in $\Omega$ associated with Galois algebra $K$, defined in reference to the basic system $\Gamma$ in $\Omega$ by

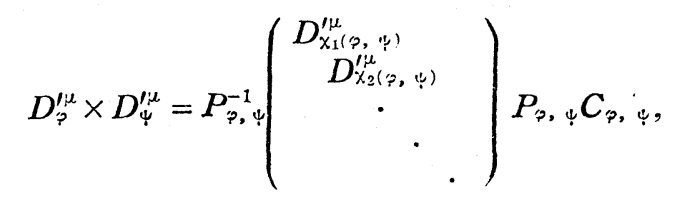

has decomposition with reference to $H$, if and only if the following conditions are satisfied; there exist a basic sysiem $\Delta=\left\{F_{r} ; \eta \in Y\right\}$ of representations of $H$ in $\Omega$ consisting of all distinct indecomposable constituents of the regular representation of $H$ over $\Omega$ and a factor system, $\{B \xi \zeta ; \xi, \zeta \in Y\}$ of $H$ in $\Omega$ such that for each representation $D_{x}$ with $\% \in X$ restricted on $H$ holds

$$
D_{x}(\tau)=\left(\begin{array}{c}
F_{\eta_{1}(\chi)}(\tau) \\
F_{r_{i}(x)}(\tau) \\
\cdot \\
\\
\end{array}\right) \text { for } \tau \in H,
$$

where $F_{r_{i}(x)}$ 's are representations belonging to $\Delta$ and with reference to the so determined correspondences $\eta_{i}(\%)$ 's between $X$ and $Y$ holds

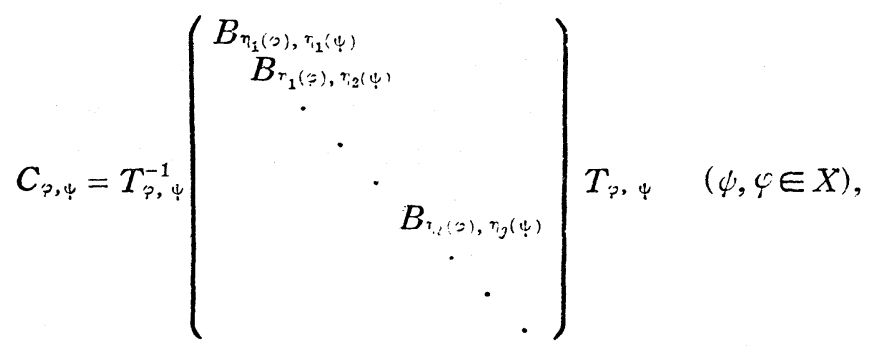

9) T. Nakayama, J. reine angew. Math. 189 (1951). 
where suffices are taken lexicographically, and $T_{\hat{\xi}, \psi}$ is a transposition matrix having 0 and 1 as its elements such that

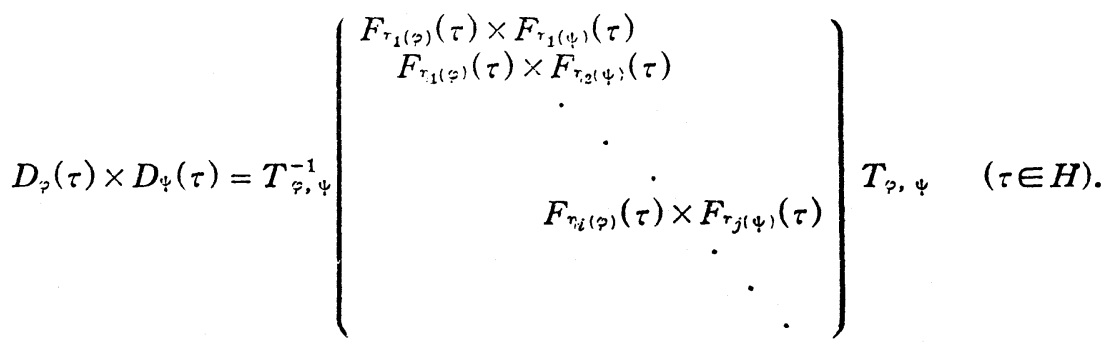

When the order of $G$ is not divisible by the characteristic of $\Omega$ and absolutely irreducible representations of $H$ are obtained in $\Omega$, the condition for a system of matrices to be a factor system, stated in $\S 1$, becomes trivial and above definition of decomposition coincides with that in my former article "Direct decompositions of Galois algebras ${ }^{10)}$ " (quoted as M. D.).

We obtain in like way as in M. D. the following theorems which generalize the main results in $M$. D..

TheoRem 1. A Galois algebra $K$ over $\Omega$ with Galois group $G$ has a decomposition with reference to $H$, if and only if there exists a factor system associated with $K$ which has a decomposition with reference to $H$.

THEOREM 2. Galois algebra $K$ over $\Omega$ with Galois group $G$ is a field if and only if there exists no factor system associated with $K$ having a decomposition with reference to a proper subgroup of $G$.

$\S 3$. Centralizer of $G$ in the group of all automorphisms of a Galois algebra.

7. Let $G$ be again an arbitrary, not necessarily finite, group and $\Re$ be an arbitrary Galois algebra obtained from $\mathfrak{M}$. Let $\rho$ denote an automorphism of the subalgebra $\mathscr{T}_{\Re}$ on itself, which is commutative with all automorphisms of $D_{\mathcal{S}}$ induced by the Galois group $G$ of $\mathscr{R}$. Then by Lemma 1 there exists for each $D_{x}^{\prime}$ one and only one matrix $C_{\varkappa}$ of degree $f_{x}$ in $\Omega$ such that

$$
D_{x}^{\prime p}=D_{x}^{\prime} C_{x}
$$

and each $C_{x}$ is, as easily seen, regular. Conversely, if an $\Omega$-permissible automorphism $\rho$ of the subalgebra $\mathscr{D}_{\Re}$ maps each $D_{x}^{\prime}$ to $D_{x}^{\prime} C_{\chi}$, where each $C_{\chi}$ is a matrix in $\Omega$, then $\rho$ is commutative with every automorphism of $\mathscr{D}_{\Omega}$ induced by the Galois group $G$. The correspondence

$$
D_{x}^{\prime} \rightarrow D_{x}^{\prime} C_{x}
$$

induces an $Q$-permissible automorphism of $D_{\widehat{A}}$ on itself, if and only if the follow-

10) K. Masuda, Tôhoku Math. J. 4 (1952). 
ing two couditions are satisfied.

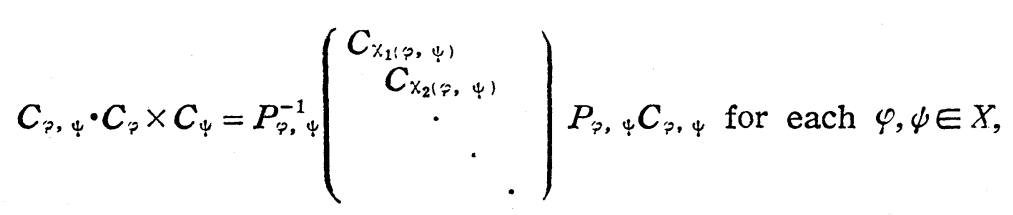

where $\left\{C_{\hat{\gamma}, \varphi} ; \varphi, \psi \in X\right\}$ is the factor system associated with $\Re$ in reference to $\Gamma$;

$$
\operatorname{Spur}\left(D_{\times}^{\prime} C_{\times} M_{\mathrm{X}}\right)=0 \quad(\% \in X)
$$

for a matrix $M_{x}$ in $\Omega$ of degree $f_{*}$, if and only if

$$
\operatorname{Spur}\left(D_{\times}^{\prime} M_{\times}\right)=0 \text {. }
$$

Let $G_{\Gamma,\{c, \psi ; \eta, \psi \in X\}}^{*}$ denote the set of all systems of matrices which satisfy the above conditions (i) and (ii). We define the product of two such systems $\left\{C_{x}^{(1)}\right.$; $\chi \in X\},\left\{C_{x}^{(2)} ; \chi \in X\right\}$ of $\left.G_{\Gamma,\left\{C_{\vartheta}, \psi\right.}^{*} \varphi, \varphi \in X\right\}$ by

$$
\left\{C_{\chi}^{(1)} ; \chi \in X\right\}\left\{C_{\chi}^{(2)} ; \chi \in X\right\}=\left\{C_{\chi}^{(1)} C_{\chi}^{(2)} ; \chi \in X\right\} .
$$

Then clearly $\left.G_{\Gamma,\{\%, \psi ;} ;, y \in x\right\}$ becomes a group, which we call the representation group of the basic system $\Gamma$ of representations of $G$ in $\Omega$ in reference to the factor system $\left\{C_{p, \psi} ; \varphi, \psi \in X\right\}$ of $G$ in $\Omega$. It is clearly isomorphic to the centralizer of the group of automorphisms of $\mathscr{D}_{\mathscr{H}}$ induced by Galois group $G$, which is isomorphic to $G$, in the group of all $\Omega$-permissible automorphisms of the subalgebra $D_{\mathscr{R}}$ of $\Re, \quad\left\{E_{f_{p} f_{\ell}} ; \varphi, \psi \subseteq X\right\}$ is clearly a factor system associated with $(\tilde{E}$, where $E_{f_{p} f_{\psi}}$ denotes the unit matrix in $\Omega$ of degree $f_{*} f_{\psi}$. For each $\sigma \in G\left\{D_{\varkappa}(\sigma)\right.$; $\sigma \in X\}$ satisfies the above conditions (i) and (ii) in reference to $\left\{E_{f_{i} f_{\psi}} ; \varphi, \psi \in X\right\rangle$,

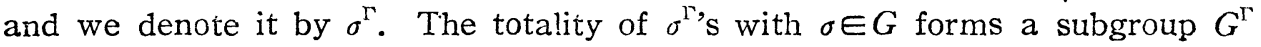

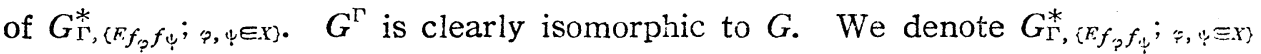
by $G_{\Gamma}^{*}$.

Now let $\S_{G}$ denote the group of all one-to-one mappings of $G$ on $G$ itself, $\sigma^{r}$ resp. $\sigma^{l}$ for each $\sigma \in G$ denote the mapping of $G$ on $G$ itself which maps each $\tau \notin G$ to $\tau \sigma$ resp. $\sigma \tau$, and $G^{r}$ resp. $G^{l}$ denote the subgroup of $\Xi_{G_{r}}$ consisting of all $\sigma^{r}$ s resp. $\sigma^{l \text { 's }}(\sigma \in G)$. As is well known, the centralizer of $G^{r}$ in $\bigodot_{G}$ is $G^{l}$, and vice versa.

8. $\Im_{G}$ may be seen as the group of all $\Omega$-permissible automorphisms of $(\dot{E}$. The group of automorphisms of (E) induced by the Galois group $G$ of $\left(E\right.$ is $G^{r}$.

When $G$ is a finite group, $\mathscr{D}_{K}$ coincides with $K$ itself, so we obtain from the above considerations the following duality for finite groups.

TheOREM 3. Let $G$ be a finite group, $\{C, \psi ; \varphi, \psi \in X\}$ be a factor system of $G$ in $\Omega$ associated with a commutative, associative and semisimple Galois algebra 
$K, H$ denote a minimal subgroup of $G$ with reference to which $K$ has a direct decomposition. Then the representation group of the basic system $I$ of representations of $G$ in $\Omega$ in reference to the factor system $\left\{C_{\varphi, \psi} ; \varphi, \psi \in X\right\}$ of $G$ in $\Omega$ is isomorphic to the centralizer of $H$ in $G$. Indeed, if the factor system $\left\{C_{\hat{\gamma}, \psi}\right.$; $\leftarrow, \psi \in X\}$ itself has a decomposition for our $H$, then the representation group of $I$ in reference to it consists of all $\left\{D_{\varkappa}(\tau) ; \% \in X\right\}$ with $\tau$ belonging to the centralizer $(5)$ of $H$ in $G$.

Proof. We prove the last part of the theorem, from which the first part follows readily. Suppose that the factor system $\left\{C_{p, \psi} ; \mathscr{\varphi}, \psi \in X\right\}$ has a decomposition with reference to $H$. Then from the fact that the centralizer of $G^{r}$ in $\Theta_{G}$ is $G^{l}$ follows that the representation group $G_{\Gamma,\left\{c_{p, \psi}^{*} ; \xi, \psi \in X\right\}}$ of $T$ with reference to $\left\{C_{\%, \psi} ; \varphi, \psi \in X\right\}$ is obtained in $G^{\Gamma}$, from the fact that $K$ is the direct sum of Galois algebras having the conjugates of $H$ in $G$ as their Galois groups follows that $G_{\Gamma,\left\{r_{r, \psi}^{*} ;, \varphi \in X\right\}}^{*}$ is contained in the normalizer of $H^{\Gamma}$ in $G^{\Gamma}$, where $H^{\Gamma}$ denotes the group consisting of all $\tau^{\Gamma}$ 's with $\tau \in H$, and from the fact that the component Galois algebras are really separable Galois fields follows that $G_{\Gamma,\left\{c_{p, \psi} ; p, \psi \in X,\right.}^{*}$ is contained in the centralizer of $H^{\Gamma}$ in $G^{\Gamma}$. Then follows easily that it coincides with the centralizer of $H^{\Gamma}$ in $G^{\Gamma}$.

If $H$ is the trivial subgroup consisting only of the unit element, then $C_{\gamma, \psi}$ $=E_{f_{y} f_{\psi}}$ and we can take in place of the condition (ii) the following weaker condition (ii)' that $C_{\chi} \neq 0(\chi \in X)$ and whenever

$$
\operatorname{Spur}\left(D_{x}^{\prime} M_{\mathrm{x}}\right)=0
$$

with a matrix $M_{x}$ in $\Omega$,

$$
\operatorname{Spur}\left(C_{\times} M_{\times}\right)=0 .
$$

It follows from the fact that the correspondence

$$
D_{x}^{\prime} \rightarrow C_{x}
$$

induces an $\Omega$-permissible homomorphism of $\xi$ in $\Omega$ if and only if $C_{\%}$ 's $(\% \in X)$ satisfy the condition (i) and (ii) ${ }^{\prime 1}{ }^{11}$

The thus obtained duality for finite groups in reference to $\left\{E_{f_{f} f_{\varphi}} ; \varphi, \dot{\varphi} \in X\right\}$ is closely related to the duality obtained by T. Nakayama in his article N. O.. Ceriain independent parameters of the linear enveloping algebra over $\Omega$ of the value matrices of representations of $G$ are obtained there with tools of the theory of modular regular representations under the restriction that absolutely irreducible representations of $G$ are obtained in $\Omega$, and the duality for finite groups is formulated in reference to the parameters, while in the present article we deal with no such independent parameters and formulate it in reference to the value

11) This remark is due to S. Takahasi. 
matrices of representations of $G$ in $\Omega$ without any restrictions on 2 , applying the condition (ii)'.

\$4. Tannaka's duality for compact $T_{0}$-groups.

9. Let, throughout $\S 4, G$ be a compact $T_{0}$-group, $\Omega$ be the field of all complex numbers, and $\Gamma=\left\{D_{\chi} ; \chi \in X\right\}$ be a unitary continuous irreducible bas.c system of representations of $G$, that is, a basic system of representation of $G$ consisting of continuous unitary irreducible representations of $G$. Let - denote either the operation to take conjugate complex numbers or an operation induced canonically by that operation. We call the subgroup $G_{\Gamma, U}^{*}$ of $G_{\Gamma}^{*}$ consisting of all systerns $\left\{C_{\times} ; \% \in X\right\}$ in $G_{\Gamma}^{*}$ with unitary $C_{\chi}$, the unitary representation group of the unitary continuous irreducible basic system $\Gamma$ of $G$. Then

$$
G_{\Gamma, U}^{*}=G_{\Gamma}^{*} \bigcap_{x \in !} U_{x,}
$$

where $U_{x}$ denotes the group of all unitary matrices of degree $f_{x}$ and $\prod_{x \in I .1} U_{\%}$ denotes the direct product group of $U_{x}^{\prime}$ 's. We denote the elements of $G_{\Gamma, U}^{*}$ by $\sigma^{*}$, $\tau^{*}$ etc., and topologize $G_{\Gamma, U}^{*}$ by the usual product topology of $\prod_{\chi \in X} U_{x}{ }^{12)}$ As the mapping $\sigma \rightarrow\left\{D_{x}(\sigma) ; \chi \in X\right\}$ gives an homeomorphism of $G^{\Gamma}$ on $G$ we identify $G^{\Gamma}$ and $G$ for brevity, that is, we denote $\sigma^{\Gamma}=\left\{D_{k}(\sigma)\right.$; $\left.\% \in X\right\}$ merely by $\sigma$. From the construction of $G_{\Gamma, U}^{*}$ we obtain for each $\% \in X$ a continuous unitary representation $D_{\chi}^{*}$ of $G_{\Gamma, U}^{*}$, if we map each $\left\{C_{\varkappa} ; \% \in X\right\}$ of $G_{\Gamma, U}^{*}$ on $C_{\%}$. It is clear that $D_{\%}^{*}(\sigma)$ $=D_{\curlywedge}(\sigma)$ for each $\sigma \in G$ and each $\psi \in X$. So each $D_{\%}^{*}(\% \in X)$ is an irreducible representation of $G_{\Gamma, t}^{*}$ and $D_{\%}^{*} \sim D_{\psi}^{*}(\varphi, \psi \in X)$, if and only if $\varphi=\psi$. Then $\left\{D_{x}^{*}\right.$; $\chi \in X\}$ is clearly a unitary continuous irreducible basic system of representations of $G_{\Gamma}^{*}, l,{ }^{13 j}$ which we denote by $\Gamma^{*}$. We define $\mathcal{F}^{*}$ and $\mathcal{D}^{*}$ for $G_{\Gamma}^{*}, \epsilon^{*}$ in reference to $\Gamma^{*}$ like $\left[*\right.$ and $\mathscr{D}$ for $G$ in reference to $\Gamma$, and denote by $\mathfrak{I}^{*}$ the closure of $\mathbb{D}^{*}$ in $c^{*}$ in the sense of uniform convergence of functions on $G_{\Gamma}^{*}, c^{*}$, that is, we define $\mathfrak{A}^{*}$ so as $f^{*} \in \mathfrak{E}^{*}$ belongs to $\mathfrak{H}^{*}$ if and only if for each given positive number $\varepsilon>0$ there exists at least a linear aggregate $\sum_{k=1}^{n} f_{\% k} \sum_{i, j=1}^{f x_{k}} a_{i, j, k} d_{i j}^{* k_{k}}$ of finite number of $d_{i j}^{* x}$ s such that

$$
f^{*}\left(\sigma^{*}\right)-\sum_{k=1}^{n} f_{\chi_{i}} \sum_{i, j=1}^{f x_{i}} a_{i, j, k} d_{i j}^{* * k}\left(\sigma^{*}\right)<\varepsilon \text { for each } \sigma^{*} \in G_{1}^{*}, v,
$$

where $f_{i_{k}}$ is the degree of $D_{r_{k}}$ and $a_{i, j, k}$ are constants. It is clear from the definition of $\mathfrak{A}^{*}$ that each $f^{*} \in \mathfrak{U}^{*}$ is a continuous almost periodic function on $G_{\mathbb{N}}^{*}, c$, that $\mathfrak{U}^{*}$ contains constants, that $\mathfrak{H}^{*}$ has sufficiently many functions

12) We do not use the compactness of either $G_{\Gamma,}^{*}$, or $\prod_{\gamma \in(i} U$.

13) $\Gamma$ resp. $\Gamma^{*}$ becomes then by Kampen's theorem a complete representative system of all conjugate classes of irreducible unitary continuous representations of $G$ resp. $G_{\Gamma, U}^{*}$. But in the present article we do not use that fact. 
on $G_{\Gamma}^{*}, c$, that is, for each different two elements $\sigma^{*}, \tau^{*} \in G_{\Gamma, v}^{*}$ there exists $f^{*} \in \mathfrak{P}^{*}$ such that

$$
f^{*}\left(\sigma^{*}\right) \neq f^{*}\left(\tau^{*}\right),
$$

and that if $\overline{f^{*}} \in \mathfrak{U}^{*}$, then $f^{*} \in \mathfrak{I}^{*}$. So we obtain the following two lemmas.

LEMma 3. If $f^{*} \in \mathfrak{R}^{*}$ maps every element of $G$ onto 0 , then it maps every element of $G_{\Gamma, v}^{*}$ onto 0 .

Proof. If $f^{*} \in \mathfrak{U}^{*}$, then clearly, from the definition of $\mathfrak{A}^{*}, f^{*}$ is a bounded function on $G_{\Gamma, v}^{*}$ and so for each positive $\varepsilon>0$ there exists $\sum_{k=1}^{n} f_{\chi_{k}} \sum_{i, j=1}^{f x_{k}} a_{i, j, k} d_{i j}^{*} x_{k}$ such that

$$
\left|\left(f^{*}\left(\sigma^{*}\right)\right)^{2}-\left(\sum_{k=1}^{m} f_{x_{i}} \sum_{i=1}^{f x_{k}} a_{i, j, k} d_{i j}^{* x_{k}}\left(\sigma^{*}\right)\right)^{2}\right|<\varepsilon \text { for each } \sigma^{*} \in G_{\Gamma, \sigma}^{*}
$$

$$
\underset{\sigma^{*} \in G_{T}^{\prime}, U}{\operatorname{Mean}}\left(\left|\sum_{k=1}^{m} f_{\chi_{k}} \sum_{i, j=1}^{f x_{k}} a_{i, j, k} d_{i j}^{* x_{k}}\left(\sigma^{*}\right)\right|^{2}\right)=\sum_{k=1}^{m} \sum_{i, j=1}^{f x_{k}}\left|a_{i, j, k}\right|^{2}
$$

we have

$$
\underset{\sigma^{*} \in G_{\Gamma, \mathrm{U}}^{*}}{\operatorname{Mean}}\left(\left|f^{*}\left(\sigma^{*}\right)\right|^{2}\right) \leqq \varepsilon+\sum_{k=1}^{m} \sum_{i, j=1}^{f x_{k}}\left|a_{i, j, k}\right|^{2}
$$

From (29) and the assumption that $f^{*}=0$ on $G$ follows

$$
\left|\sum_{k=1}^{m} f_{x_{k}} \sum_{i, j=1}^{f x_{k}} a_{i, j, k} d_{i j}^{X_{k}}(\sigma)\right|^{2} \leqq \varepsilon \text { for each } \sigma \in G .
$$

As it is clear that

$$
\underset{\sigma \in(i}{\operatorname{Mean}}\left(\left|\sum_{k=1}^{m} f_{x_{i}} \sum_{i, j=1}^{f x_{k}} a_{i, j, k} d_{i j}^{X_{k}}(\sigma)\right|^{2}\right)=\sum_{k=1}^{m} \sum_{i, j=1}^{f x_{k}}\left|a_{i, j, k}\right|^{2},
$$

we have from (32)

$$
\sum_{k=1}^{m} \sum_{i, j=1}^{f x_{k}}\left|a_{i, j, k}\right|^{2} \leqq \varepsilon,
$$

whence

$$
\underset{\sigma^{*} \in G_{\Gamma, U}^{*}}{\operatorname{Mean}}\left(\left|f^{*}\left(\sigma^{*}\right)\right|^{2}\right) \leqq 2 \varepsilon .
$$

Let'sing $\varepsilon$ tend to 0 , we obtain

$$
\underset{\sigma^{*} \in G_{\Gamma}^{*}, U}{\operatorname{Mean}}\left(\left|f^{*}\left(\sigma^{*}\right)\right|^{2}\right)=0,
$$

which implies

$$
f^{*}\left(\sigma^{*}\right)=0 \text { for every } \sigma^{*} \in G^{*}, \quad \text { q.e.d. }
$$


We denote by $\mathfrak{i}$ the subring of $\mathbb{E}$ which consists of all mappings of $G$ into $\Omega$ given by $f^{*} \in \mathfrak{H}^{*}{ }^{*}{ }^{\mathrm{l}}$, Then

Lemma 4. Every proper ideal $\mathfrak{P}$ of $\mathfrak{P}$ has zero, that is, there exists $\sigma \in G$ such that

$$
f(\sigma)=0
$$

for all $f \in \mathbb{P}$.

Proof. Suppose that an ideal $\mathfrak{P}$ does not possess a zero. Since $G$ is compact, there exists then a finite set of elements $f_{1}, f_{2}, \ldots, f_{r} \in \mathfrak{P}$ and a positive number $\varepsilon>0$ such that

$$
\sum_{i=1}^{n} f_{i}(\sigma) \overline{f_{i}(o)} \gg \varepsilon>0 \quad \text { for each } \quad \sigma \in G .
$$

From the definition of $\mathfrak{A}$, there exist $f_{1}^{*}, f_{2}^{*}, \ldots, f_{r}^{*}$ such that

$$
f_{i}^{*}(\sigma)=f_{i}(\sigma) \text { for every } \sigma \in G \text {. }
$$

Then $\sum_{i=1}^{r} f_{i}^{*} f_{i}^{*}$ is a non-negative real function on $G_{\Gamma, U}^{*}$ and indeed

$$
\sum_{i=1}^{r} f_{i}^{*}(\sigma) \overline{f_{i}^{*}(\sigma)} \geq \varepsilon>0 \text { for every } \sigma \in G .
$$

Put

$$
\stackrel{\varphi}{*}^{*}\left(\sigma^{*}\right)=\operatorname{Max}\left(\sum_{i=1}^{r} f_{i}^{*}\left(\sigma^{*}\right) f_{i}^{*}\left(\sigma^{*}\right), \frac{\varepsilon}{2}\right) .
$$

Then $\varphi^{*}\left(\sigma^{*}\right)$ can be approximated by polynomials of $\sum_{i=1}^{r} f_{i}^{*} f_{i}^{*}{ }^{*}$ ) So

$$
\varphi^{*} \in \mathfrak{T}^{*} \text {. }
$$

As

$$
\varphi^{*}\left(\sigma^{*}\right) \gtrsim \frac{\varepsilon}{2}>0 \text { for every } \sigma^{*} \in G_{\Gamma, t}^{*},
$$

$1 / \mathcal{C}^{*}\left(\sigma^{*}\right)$ is a continuous function and can be approximated by polynomials of $\varphi^{*}\left(\sigma^{*}\right),{ }^{15)}$ whence $1 / c^{* *}\left(\sigma^{*}\right) \in \mathfrak{2} *$. So we have

$$
1 / \varphi \in \mathfrak{A}
$$

14) We do not explicitly deal with the totality of continuous almost periodic functions of either $G_{\Gamma, G}^{*}$ or $G$, though $\mathfrak{H}^{*}$ resp. $\mathfrak{A}$ coincides really that of $G_{\Gamma, E}^{*}$ resp. $G$.

15) Here we need apply only classical Weierstrass approximation theorem to concretely defined two real functions of a real variable. But we need apply Neumann's generalized one and Urysohn's existence theorem of continuous functions to prove the existence of sufficiently many unitary continuous representations of $G$, which we supposed in the present article already in $\S 1$, No. 3. 
where we denote by $1 / \varphi$ the mapping of $G$ into $\Omega$ induced by $1 / \varphi^{*}$ restricted on $G$. Then, as clearly

$$
1 / \varphi(\sigma) \sum_{i=1}^{r} f_{i}(\sigma) \overline{f_{i}(\sigma)}=1 \text { for every } \sigma \in G
$$

$$
\mathfrak{P} \ni 1 \text {, }
$$

which proves the lemma.

From the above Lemmas Tannaka's duality follows:

Theorem (Tannaka).

$$
G_{\Gamma, U}^{*}=G^{\Gamma}
$$

Proof. Suppose $G_{\Gamma, v}^{*} G^{\Gamma}$. We take $\sigma_{0}^{*}$ such that

$$
\sigma_{0}^{*} \in G_{\Gamma, U}^{*}, \quad \sigma_{0}^{*} \in G^{\Gamma} .
$$

Let $\mathfrak{P}^{*}$ be the maximal ideal of $\mathfrak{A}^{*}$ consisting of all functions of $\mathfrak{H}^{*}$ with 0 as their value at $\sigma_{0}^{*}$, and $\mathfrak{P}$ be the ideal of $\mathfrak{X}$ consisting of all mappings of $G$ into $\Omega$ given by the elements of $\mathfrak{B}^{*}$. Then clearly

$$
\mathfrak{A}^{*} / \mathfrak{P}^{*} \cong \Omega
$$

and it follows from Lemma 3 that

$$
\mathfrak{H}^{*} \cong \mathfrak{H}, \quad \mathfrak{P}^{*} / \mathfrak{P}^{*} \cong \mathfrak{Y} / \mathfrak{P}(\cong \Omega) .
$$

By Lemma $4 \mathfrak{B}$ has a zero in $G$. This contradicts the fact that $\mathfrak{H}^{*}$ contains sufficiently many functions on $G_{\Gamma}^{*}, v$. So we must have

$$
G_{\Gamma, U}^{*}=G^{\Gamma} .
$$

Departinent of Mathematics

Yamagaia University 